\title{
TRASTORNOS MUSCULOESQUELÉTICOS EN EL PERSONAL DE UNA EPS EN LA CIUDAD DE CALI, PERIODO 2013-2015
}

\author{
Astrid Ordóñez, Juliet Restrepo y Claudia Castañeda. \\ Semillero SERGOS \\ Grupo de investigación GISAP \\ Institución Universitaria Antonio José Camacho \\ Recibido: 03/03/2020. Aprobado: 12/06/2020 \\ Cómo citar este artículo: \\ Ordóñez, A., Restrepo, J. y Castañeda, C. (2020). Trastornos musculoesqueléticos en el personal de una EPS en la ciudad \\ de Cali, periodo 2013-2015. Revista Sapientía, 12 (24), 27-35.
}

\section{RESUMEN}

Es un estudio descriptivo cuantitativo y cualitativo con un enfoque retrospectivo, el objetivo es identificar los factores biomecánicos que pueden ocasionar trastornos musculoesqueléticos en el personal administrativo de una Entidad Promotora de Salud de la ciudad de Cali en el periodo 2013-2015.

Las estadísticas de La OIT informan que las enfermedades profesionales ocasionan un número de ausentismo seis veces mayor que los accidentes laborales, por ende, el 2.02 millones son causados por enfermedades de trastornos musculoesqueléticos (TME) relacionados con el trabajo, estimando una pérdida del $4 \%$ del producto interno bruto (PIB). Para el Instituto Nacional para la Seguridad y Salud Ocupacional (NIOSH) de los Estados Unidos, el sector manufacturero es el más afectado por los TME con 41/1000 empleados. En Colombia, el informe realizado por el Ministerio de Protección Social afirma que, en el año 2001, con 27\%, el síndrome de túnel carpiano era la primera causa de morbilidad profesional, con tendencia a acrecentar. Según FASECOLDA, con base en datos de la segunda Encuesta Nacional de Condiciones de Seguridad y Salud en el Trabajo en el Sistema de Riesgo, para el 2012 de 40.000 enfermedades laborales, el 88\% de estas patologías fueron TME, con predominio del síndrome del túnel del carpo.

En ciudades como Bahía (Brasil) y Morelia (México), en el año 2010, se realizaron estudios acerca de los factores asociados a los trastornos musculoesqueléticos (TME) que evidenciaron los factores biomecánicos como los causantes de ausentismo, con un indicador de morbilidad alto.

Para este trabajo se tomó una muestra de 68 trabajadores por medio de una fórmula, con una población de 270 trabajadores, aplicando criterios de inclusión y exclusión, usando instrumentos de asociación por objetivos específicos (base de ausentismo, método RULA, método estadístico univariado y bivariado).

Los resultados permitieron identificar que el género femenino, entre 20 a 30 años, y el género masculino, entre 40 a 45 años, son la población más afectada a causa de los trastornos musculoesquelético (TME) por factores biomecánicos. 


\section{PALABRAS CLAVES}

Biomecánico, Factores, Trabajador, Trastornos, Musculoesquelético, Universalidad, Articulaciones, Ocupaciones, Estadística, método, RULA, confidencialidad.

\section{ABSTRACT}

It is a quantitative and qualitative descriptive study with a retrospective approach, the aim is to identify biomechanical factors that can cause musculoskeletal disorders in the administrative staff of a promoter of health city Cali in the 2013-2015 period.

OTI statistics report that occupational diseases cause a number of absenteeism six times greater than occupational accidents, therefore 2.02 million are caused by diseases of work-related musculoskeletal disorders (SMD), estimating a loss of $4 \%$ of the gross domestic product (GDP). For the National Institute for Occupational Safety and Health (NIOSH) of the United States, the manufacturing sector is the most affected by the TME with 41/1000 employees. In Colombia, the report carried out by the Ministry of Social Protection affirms that carpal tunnel syndrome was the first cause of professional morbidity in $27 \%$ in 2001 with a tendency to increase. According to FASECOLDA, more than 40,000 occupational diseases, according to the second National survey of Occupational Safety and Health Conditions in the risk system for $2012,88 \%$ of these pathologies were the (TME) prevailing tunnel syndrome. carpus.

Countries such as Bahia, Brazil and Morelia, Mexico in 2010 conducted a study about the factors associated with disorders (MSDS) skeletal muscle, where it showed that biomechanical factors were the cause of absenteeism, with an indicator of morbidity Alto.

Took a sample of 68 workers by means of a formula, with a population of 270 workers applying inclusion and exclusion criteria, using instruments of Association by specific objective (method RULA, based on truancy, statistical method Univariate and bivariate).

This work identified in its results that feminine gender was the population most affected between 20 to 28 years of age because of disorders (MSDS) skeletal muscle by biomechanical factors and the masculine gender of 40 to 45 years of age.

\section{KEYWORDS}

Biomechanical Factors, Worker, Disorders, Musculo-Skeletal, Universality, Joints, Occupations, Statistics, Method, RULA, Confidentiality.

\section{INTRODUCCIÓN}

Las enfermedades profesionales están contempladas y definidas por la OIT como "las que se saben provenientes de la exposición a sustancias o condiciones peligrosas inherentes a ciertos procesos, oficios u ocupaciones" (OIT, 2010). La OIT (2010) también define los trastornos musculoesqueléticos como "cualquier daño o trastorno de las articulaciones y otros tejidos, debido a movimientos repetitivos, esfuerzos intensos, vibraciones y posturas extremas". Las estadísticas de la OIT reportan que las enfermedades profesionales ocasionan un número de muertes seis veces mayor que los accidentes laborales, de 2.34 millones de muertes anuales relacionadas con el trabajo, de las cuales 2.02 millones son causadas por enfermedades de trastornos musculoesqueléticos relacionados con el trabajo (OIT. EP, 2013). En la Unión Europea cada año más de 5.850 personas pierden su vida como consecuencia de los accidentes de trabajo $y$ otros 159.000 fallecen a causa de enfermedades profesionales (EU. OSHA, 2012).

La evaluación encontrada por el Instituto Nacional para la Seguridad y Salud Ocupacional de los Estados Unidos (NIOSH) reporta una alta tasa de prevalencia de síntomas de trastornos 
musculoesqueléticos asociados al trabajo evaluado, presentando una afectación en espalda inferior (80.2\%), manos y muñecas (48.4\%), hernias discales (14\%), síndrome del túnel carpiano (16\%), inflamación de tendones (tendinitis), revestimiento de las vainas que cubren el tendón (tenosinovitis), bursitis, distensión muscular y patologías en los nervios de extremidades superiores, el cuello y la espalda. En California, el sector agrícola reporta más de 3.000 trabajadores con lesiones en espalda con un costo estimado de más de $\$ 22$ millones de dólares al año en solo compensaciones para trabajadores, aunque puede haber muchas lesiones que no se reportan (NIOSH, 2012).

En 2014 en Honduras se realizó un estudio basado en los trastornos musculoesqueléticos y psíquicos en la población trabajadora de maquila de la confección, resultado del cual encontraron que el $78 \%$ de los empleados sufren de trastornos musculoesqueléticos y el $45.6 \%$ sufren de trastornos psiquiátricos, debido a las jornadas extenuantes a las que se ven sometidos (Pérez \& Martínez, 2014). En Ecuador se realizó un estudio sobre los síntomas musculoesqueléticos en trabajadores operativos del área de mantenimiento de una empresa petrolera ecuatoriana, cuyo objetivo era determinar la prevalencia de los síntomas; los resultados indicaron que la mayor prevalencia de síntomas musculoesqueléticos se encuentran en un grupo de trabajadores entre los 30 y 40 años de edad, en regiones anatómicas como: espalda baja $(64.7 \%)$, espalda alta $(43.1 \%)$, cuello (37.3\%), hombro (26.5\%), con mayor afectación en los puestos de trabajo de las áreas técnico-eléctrico y técnico-mecánico (Águila et al, 2014).

En Colombia, la Federación de Aseguradores Colombianos (FASECOLDA) informa que en el 2018 se presentaron 645.119 accidentes laborales, con una disminución del $2.3 \%$ a comparación del 2017; el análisis resalta que las enfermedades calificadas tuvieron un aumento del 7.1\% (para un total de 10.435) y respeto a la mortalidad el aumento no fue significativo, sin embargo, para el 2018 se presentaron 569 muertes de origen laboral. En cuanto a la accidentalidad laboral, FASECOLDA no especifica la causa, pero sí identifica los sectores económicos de mayor afección, clasificándolos de la siguiente manera: en primer lugar, el sector minas y cantera con una tasa de 13 accidentes por cada 100 trabajadores afiliados al sistema de riesgos laborales; en segundo lugar, el sector de agricultura, ganadería, caza y silvicultura, con una tasa de 12 accidentes por cada 100 trabajadores. Para FASECOLDA las enfermedades laborales tuvieron un incremento significativo en el sector económico de servicios domésticos con un 57\%, la pesca con $24 \%$, hoteles y restaurantes con el $23 \%$, sin embargo, siguen a la cabeza el sector económico de minas y canteras con un aumento del $10 \%$. Asimismo, el análisis comparativo entre el año 2017 versus 2018 indica que el departamento con mayor accidentalidad y enfermedad laboral es el Departamento de Magdalena con un aumento del $4 \%$, seguido del Departamento de Cundinamarca, quien presentó una disminución del 6\% (Consejo Colombiano de Seguridad, 2018).

El presente artículo busca identificar si el factor biomecánico es el causante de los trastornos musculoesqueléticos en el personal administrativo, específicamente en el periodo del año 2013, donde los trastornos musculoesqueléticos estaban representados por un $4.96 \%$ y entre el periodo 2014-2015 por un 5.84\% de la población afectada. A partir de lo cual se encuentra que el ausentismo por lesiones musculoesqueléticas se presenta con mayor prevalencia en el personal administrativo femenino con un $5.6 \%$, con una diferencia no muy marcada de $5.2 \%$ en hombres, según la base de ausentismo suministrada por la entidad objeto estudio. Asimismo, se identifican los tipos de lesiones ocasionadas a saber: traumatismos en pie y tobillo, tendinitis del bíceps, traumatismo del tendón del manguito rotador, síndrome del manguito rotatorio, fracturas del diáfisis del radio, cervicalgia, lumbalgias, dolor en articulaciones, sinovitis, tendosinovitis, trastornos sinovial, tendinosis, contracturas de tendón (vaina), fracturas de los dedos de la mano, lumbago con ciática, esguinces $y$ torcedura de muñeca, trastornos del disco 
lumbar con radiculopatía, trastornos de los discos intervertebrales, epicondilitis, traumatismos de estructuras múltiples de rodilla, esguince de tobillo, fracturas de vertebras lumbar, rupturas traumáticas de ligamentos de los dedos de las manos, tendinitis rotuliana, luxación del codo, amputación de mano derecha e incidentes leves por deslizamiento (Daruma Coomeva EPS, 2015).

El aporte de este estudio consiste en suministrar a la empresa una alerta acerca del riesgo biomecánico al que está expuesto el personal administrativo y el correspondiente el ausentismo ocasionado por trastornos musculoesqueléticos que afecta al género femenino en el rango de edad entre 20 y 30 años y al género masculino entre los 40 y 45 . De igual manera, el conocimiento resultante de este trabajo pretende servir de ayuda didáctica para otros profesionales que quieran hacer un estudio sobre esta problemática y, en esa misma medida, contribuir al área de estudio.

\section{MARCO TEÓRICO}

El sistema musculo esquelético está compuesto por una serie de músculos, tendones y huesos, cuya función es efectuar movimientos y esfuerzos necesarios para la vida. El sistema está sostenido por la columna vertebral, que en su interior alberga la médula espinal, conectora del sistema nervioso central y el sistema nervioso periférico (Mondelo, Gregori, \& Barrau, 2000). Según Luttmann (et al, 2004), existe un nexo causal entre los trastornos musculoesqueléticos y el esfuerzo físico realizado durante la actividad laboral; la dolencia y lesiones que efectúan los músculos, tendones, articulaciones, ligamentos y huesos son causados principalmente por un esfuerzo mecánico excesivo de las estructuras biológicas.

En Colombia, con base en los resultados de los informes descritos por el Ministerio de Protección Social, se definió un Plan Nacional de Prevención de Desórdenes Musculoesqueléticos generando una guía para el desarrollo de un sistema de vigilancia epidemiológica para la prevención de los desórdenes musculoesqueléticos. La dirección general del riesgo profesional del Ministerio de Protección Social publicó en el año 2004 el Informe de las Enfermedades Profesionales en Colombia, el cual afirmó que del 2001 al 2005 el 22\% de los diagnósticos de síndrome de conducto carpiano eran la primera causa de morbilidad profesional; la segunda causa, con 9\%, eran los trastornos de disco vertebral (Tafur Sacipa \& Giraldo Suárez, 2006).

Entre el año 2009 y 2013 se calificaron en Colombia, según la Federación de Aseguradores Colombianos (FASECOLDA), más de 40.000 enfermedades laborales. De acuerdo con la Segunda Encuesta Nacional de Condiciones de Seguridad y Salud en el Trabajo, en el sistema de riesgo para el 2012, el $88 \%$ de estas patologías fueron desórdenes musculoesqueléticos (DME), entre las que se encuentran enfermedades como el síndrome del túnel carpiano y las tendinitis que se producen en las manos, codos y hombros, y los problemas de espalda, tales como el lumbago; las enfermedades auditivas, respiratorias, relacionadas con el estrés y otros trastornos ocupan el $12 \%$. En 2009, la compañía de seguros administradora de riesgos laborales Positiva hizo un análisis donde identificó las necesidades más importantes para prevenir e intervenir sobre las diferentes enfermedades laborales calificadas, ya que el $90 \%$ de las mismas están relacionadas con problemas musculoesqueléticos. La estrategia consistió en direccionar los programas y en desarrollar diferentes propuestas para la prevención que pueden adaptarse a las necesidades de la empresa, a su sector económico y tamaño. En las 276 empresas donde se está implementando esta estrategia se ha evidenciado una reducción sustancial en la tasa de ausentismo por DME y en la incidencia de este tipo de enfermedades (FASECOLDA, 2016).

\section{METOdOLOGÍA}

El tipo de estudio es descriptivo transversal cuantitativo y cualitativo de los datos obtenidos, 
con un análisis retrospectivo de la información sustraída de los informes de ausentismos y diagnósticos de la población objeto de estudio de una entidad promotora de la ciudad de Cali durante los años 2013-2015, con una población de 270 trabajadores para la ejecución de cada uno de los métodos: RULA, base de ausentismo, método univariado y bivariado.

A la población objeto de estudio, constituida por 68 trabajadores, se le aplicó criterios de inclusión y exclusión, y el método RULA. La muestra fue determinada por un nivel de confianza del 95\%. El estudio sirve para determinar estadísticamente el porcentaje de aparición de un determinado suceso; la estadística no resuelve problemas, los identifica y señala la solución.

Para dar cumplimiento al objetivo propuesto en el estudio se requiere de la recolección de la información por medio de los instrumentos metodológicos tales como la hoja de campo para recolección de datos utilizada en el método RULA, registro fílmicos y fotográficos de la ejecución de las tareas y la base de datos de ausentismo suministrada por la entidad a estudio, y el método estadístico univariado y bivariado para el análisis de los datos obtenidos.

\section{RESULTADOS}

En los resultados de la caracterización de todos los puestos de trabajo de la base de datos del ausentismo, para el año 2013, se halló que el género femenino fue el más afectado por los factores biomecánicos en un rango de edad entre 23 a 55 años, con un rango de ausentismo entre 212 a 282 días en el periodo de estudio. En cuanto a la representación de este factor en el género masculino, en el rango de edades entre 28 a 58 años, el tiempo de ausentismo es bajo comparativamente con el género opuesto, ya que oscila entre un rango de 67 a 134 días en el mismo periodo. Ver figuras 1 a la 3.

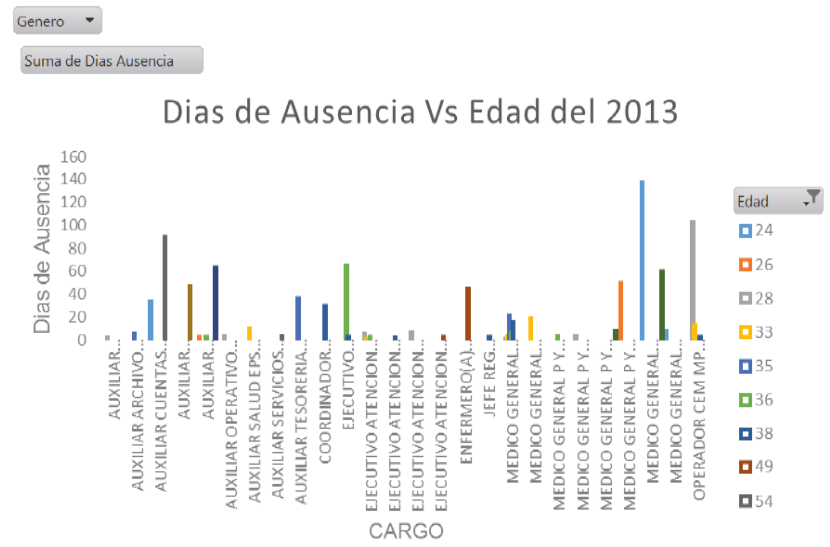

Cargo -

Figura 1. Días de ausentismo/ edad año 2013.

Fuente: elaboración propia

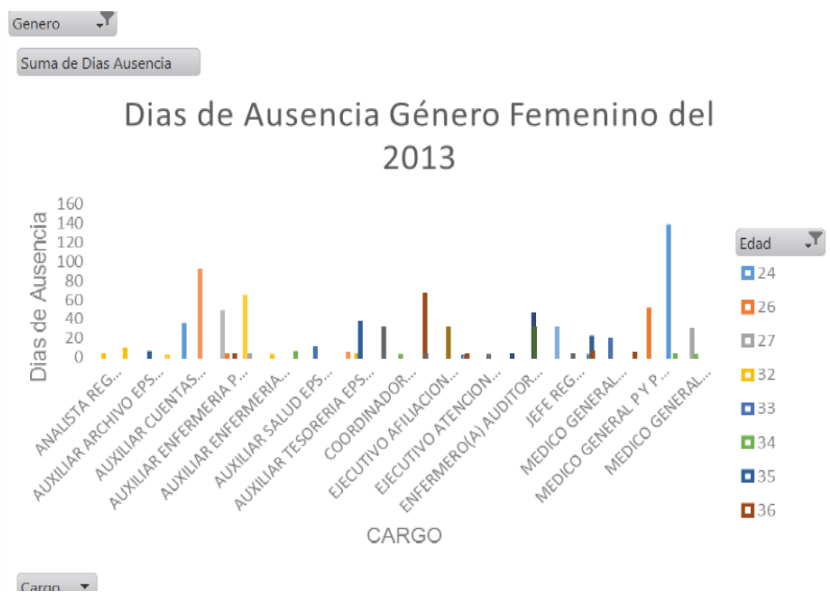

Figura 2. Ausentismo por Cargo Vs Edad, género femenino 2013.

Fuente: elaboración propia

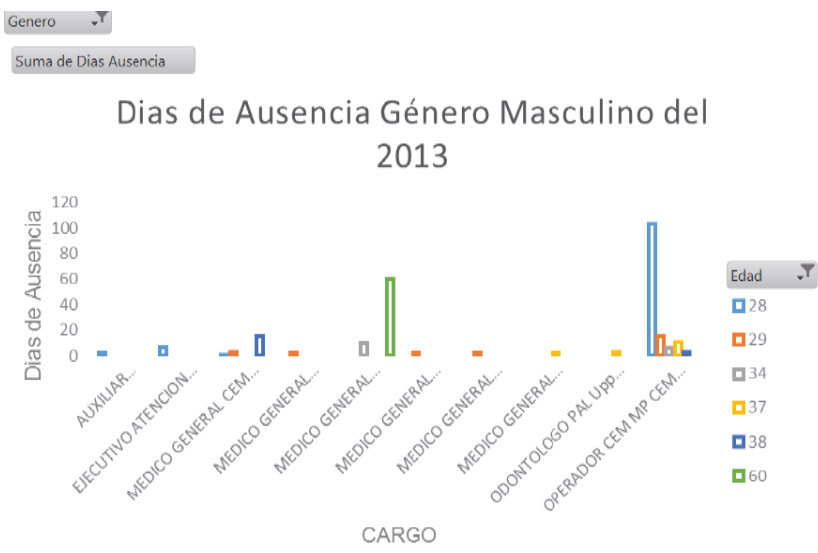

Figura 3. Ausentismo por Cargo Vs

Edad, género masculino en el año 2013.

Fuente: elaboración propia 
Los resultados obtenidos por la caracterización en la base de ausentismo, con relación a los trastornos musculoesqueléticos, arrojaron que el $16 \%$ presenta signos de síndrome de manguito rotador; el $14 \%$ diagnósticos de esguinces; el 13\% torceduras de dedos de las manos y lumbalgia, independiente del género. Estos son los síntomas más representativos que ocasionaron los días de ausentismo anteriormente mencionados. Ver figuras 4 y 5 .

\section{Transtorno Músculo-esqueléticos en el 2013}

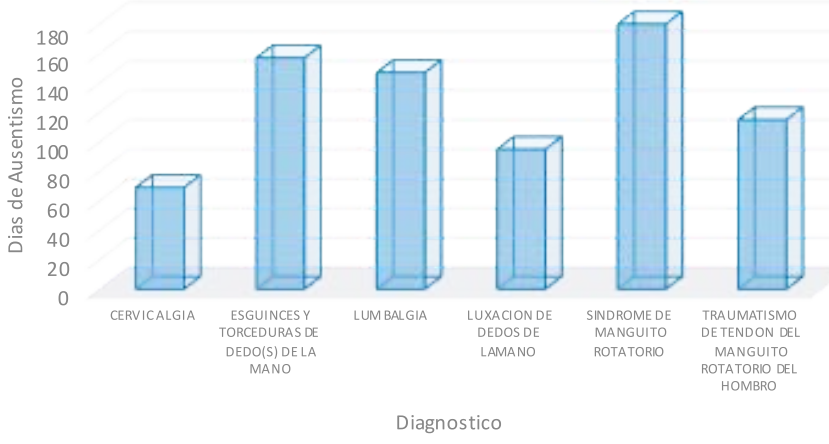

Figura 4. Ausentismo por trastornos musculoesqueléticos en el año 2013.

Fuente: elaboración propia

Trastornos Músculo-Esquelético en el año 2013

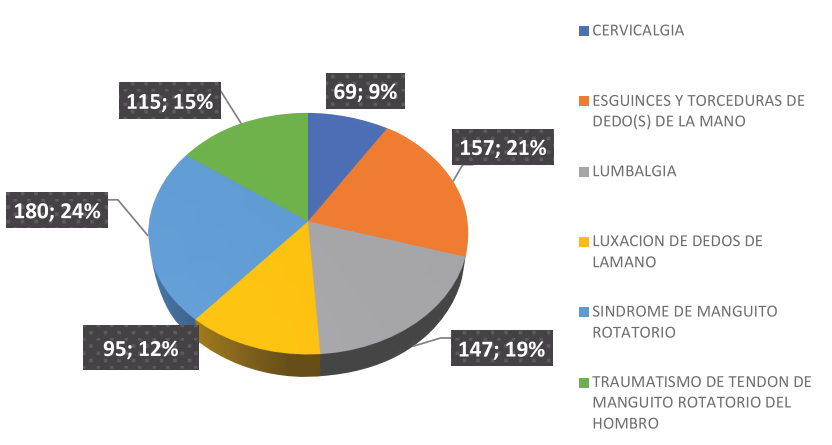

Figura 5. Ausentismo por trastornos musculoesqueléticos en el año 2013.

Fuente: elaboración propia

La caracterización que se realizó a la base de ausentismo de los años 2014 y 2015 no evidencia ninguna particularidad en la mejora de la prevención de los síntomas. Se sigue observando que el género femenino es el más afectado, no varía en sus días de ausentismo, ni en el rango de edad mencionada al principio de la identificación del resultado, continuando la afectación con diagnósticos como el lumbago (22\%) y las fracturas de los dedos de las manos (12\%). Ver figuras 6 y 7.

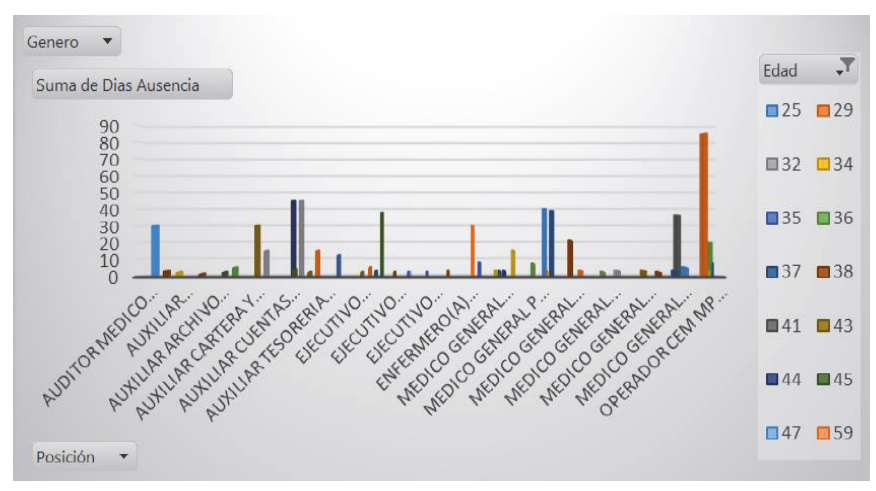

Figura 6. Días de ausentismo/ edad año 2014-2015.

Fuente: elaboración propia

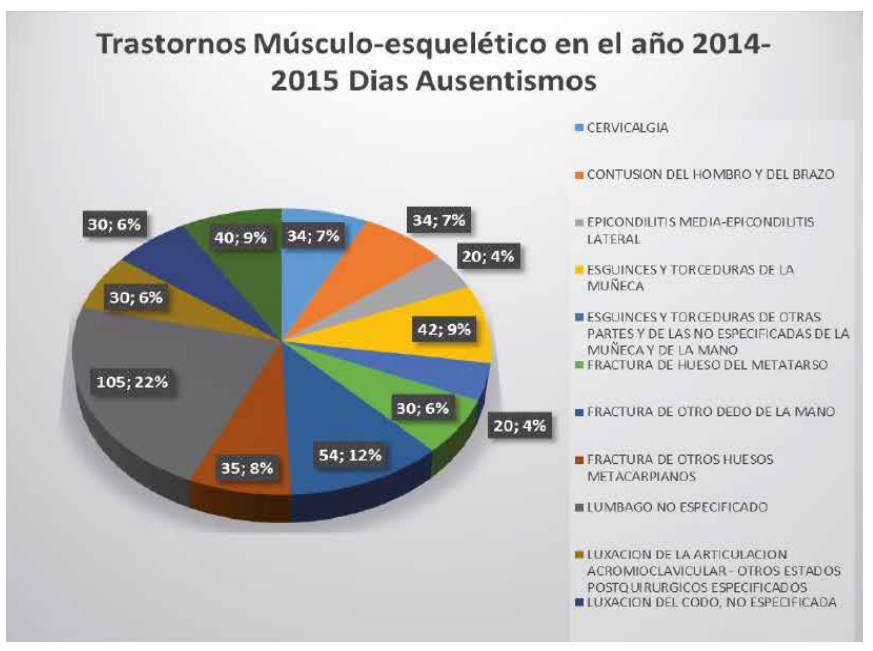

Figura 7. Ausentismo por trastornos musculoesqueléticos. Año 2014-2015.

Fuente: elaboración propia

Los resultados obtenidos por el método RULA a través de mediciones de ángulos de los miembros superiores (antebrazo, brazo y muñeca) y miembros inferiores (cuello, tronco, piernas) permitieron definir que los factores biomecánicos serían la causa principal de la manifestación de los trastornos musculoesqueléticos. Teóricamente, el método permite realizar mediciones de ángulos y posturas por medio de herramientas fotográficas que se tomaron a 68 trabajadores de la población muestra; 
este método permite evaluar una puntuación del 1 al 7 con un nivel de actuación del riesgo del 1 al 4 , siendo el 4 el nivel de actuación más alto y el 7 la puntuación más alta representando la vulnerabilidad. En las siguientes figuras se representa el resultado del método, según el cual se halla que la puntuación y el nivel de actuación en los miembros superiores (brazo, antebrazo, muñeca) representan un riesgo de 7 para la población objeto de estudio con un $77 \%$ de mayor vulnerabilidad. Ver figura 8.

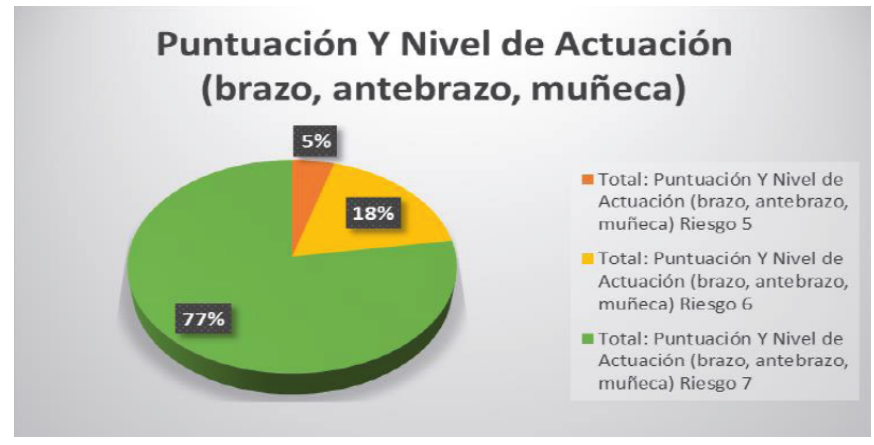

Figura 8. Puntuación y nivel de actuación (brazo, antebrazo, muñeca).

Fuente: elaboración propia

La puntuación y el nivel de actuación en los miembros inferiores (cuello, tronco, piernas) constituyen un riesgo de 4 , equivalente a un $46 \%$ de la población objeto de estudio con una baja vulnerabilidad. Por lo que los miembros inferiores constituyen la zona menos afectada por los factores biomecánicos (ver figura 9).

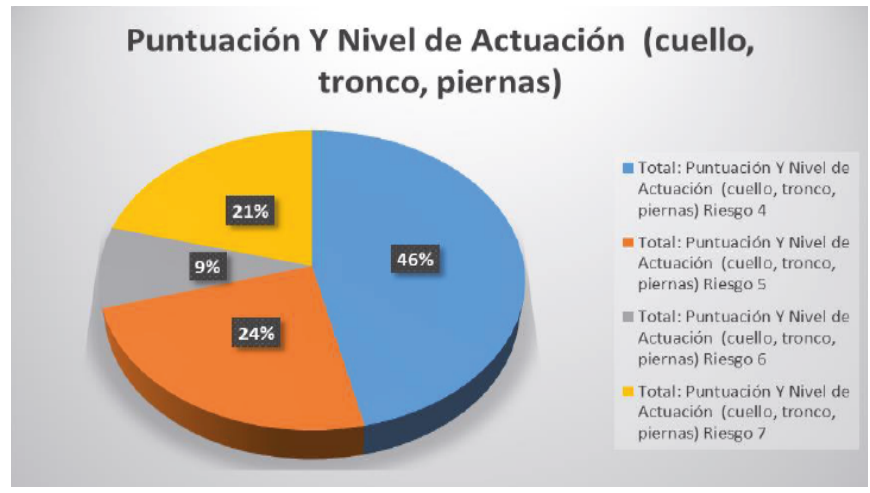

Figura 9. Puntuación y nivel de actuación (cuello, tronco, piernas).

Fuente: elaboración propia
El análisis estadístico descriptivo univariado y bivariado se realiza con el fin de identificar la relación entre los factores biomecánicos y los trastornos musculoesqueléticos. En este orden de ideas se analizan las variables de edad, posturas de miembros superiores e inferiores y ausentismos, extraídos de los resultados de la base de ausentismos y los resultados del método RULA. Para el análisis univariado se usaron las siguientes variables: edad, ausentismos y grupos diagnósticos, hallando que durante los años 2013 a 2015 el rango de edad más afectada está entre 25 y 45 años y la parte de mayor afectación es el tronco con un 20,59\%, seguida de la muñeca con un $19,12 \%$ y el brazo con el $13,24 \%$, representando entre 3 a 7 días de ausentismo por trabajador. Ver figuras 10 y 11.

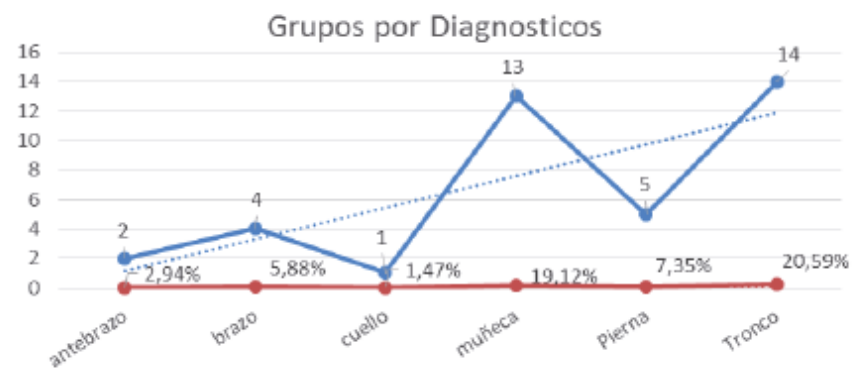

Figura 10. Análisis estadístico descriptivo univariado. Grupos por diagnósticos.

Fuente: elaboración propia

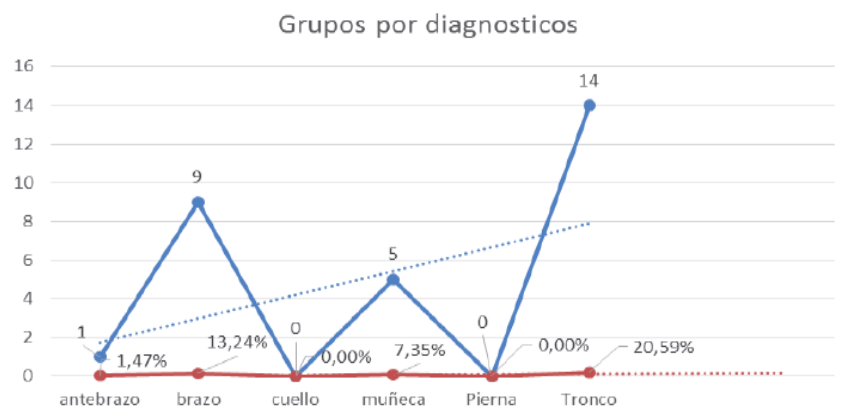

Figura 11. Análisis estadístico descriptivo univariado. Grupos por diagnósticos.

Fuente: elaboración propia

Para el resultado del análisis estadístico bivariado se usó la recopilación de las variables de edad, ausentismo y diagnóstico del análisis univariado, que no presenta un cambio marcado, pero sí ratifica el rango de edad afectada por el riesgo biomecánico 
causante de los trastornos musculoesqueléticos que ocasionan el mismo tiempo de días de ausentismo. en edades entre 24 y 30 años, por trastornos musculoesqueléticos por factores biomecánicos.

\section{CONCLUSIONES}

Por medio de la caracterización de la base de ausentismo se halló que, entre los años 2013 y 2015, el género femenino fue el más afectado por los trastornos musculoesqueléticos originados por factores biomecánicos, en un rango de edad entre 24 a 30 años, con un ausentismo de 289 días en total.

Para identificar si el factor biomecánico es el causante de los trastornos musculoesqueléticos del personal objeto de estudio se usó la herramienta método RULA, como instrumento para la valoración y medición de los ángulos posturales, evaluando así la exposición de los factores de riesgo que arrojan como resultado un alto nivel de riesgo (nivel 7) hacia el género femenino afectado. Estas mediciones permiten una revisión de tareas para mejorar las causas que están generando los trastornos musculoesqueléticos que son la mayor causa de ausentismo. A partir de lo cual se determina como tareas de mejora: implementar la cultura de higiene postural en general; realizar una evaluación ergonómica de puestos de trabajo para determinar si se requiere rediseño del puesto $y$, finalmente, hacer revisión de las mediciones de iluminación en los puestos de trabajo para verificar su buen desempeño, ya que este es un factor que conlleva a un colaborador a una mala higiene postural.

El análisis estadístico descriptivo univariado y bivariado permite familiarizar variables como edad, ausentismo, postura, género, frecuencia, prevalencia e incidencia que conllevan a los riesgos biomecánicos y, finalmente, derivan en trastornos musculoesqueléticos. Este análisis nos permite además asociar la variable y los resultados del método RULA (posturas, medición de ángulos) para determinar la variable afectada por su exposición $\mathrm{y}$ efecto, mostrando que el género femenino es el más afectado con una frecuencia y prevalencia

\section{REFERENCIAS BIBLIOGRÁFICAS}

Agila-Palacios, Enmanuel, Colunga-Rodríguez, Cecilia, González-Muñoz, Elvia, \& Delgado-García, Diemen. (2014). Síntomas Musculoesqueléticos en Trabajadores Operativos del Área de Mantenimiento de una Empresa Petrolera Ecuatoriana. Ciencia \& trabajo, 16(51), 198-205. https://dx.doi.org/10.4067/ S0718-24492014000300012

Consejo Colombiano de Seguridad. (2018). Cómo le fue a Colombia en accidentalidad, enfermedad y muerte laboral en 2018. Recuperado de https://ccs. org.co/como-le-fue-a-colombia-en-accidentalidadenfermedad-y-muerte-laboral-en-2018/

FASECOLDA, F. (2016). FASECOLDA en Acción. Recuperado el 29 de Julio de 2014, de http://www. FASECOLDA.com/index.php/sala-de-prensa/ noticias/2014/accion-julio-29-2014/

Luttmann, A. P., Jager, M. P.-I., Griefahn, B. P., Caffier, G. D., \& Lieberts, F. D. (2004). Prevención de trastornos musculoesqueléticos en el lugar de trabajo. En J. C. Mossink, \& N. Deborah Imel (Ed.). Información sobre factores de riesgos y medidas preventivas para empresarios, delegados y formadores en salud laboral. Recuperado de http://cdrwww.who.int/ occupational_health/publications/en/pwh5sp.pdf.

Ministerio de Salud y Protección Social. (2015). Enfermedad laboral. Bogotá: Ministerio de Salud y Protección Social. Recuperado de https://www. minsalud.gov.co

Ministerio de la Protección Social. (2006). Guías de Atención Integral Basada en la Evidencia para Desórdenes Musculoesqueléticos (DME) relacionados con movimientos repetitivos de miembros superiores (síndrome del túnel del carpo, epicondilitis y enfermedad de Quervain) (GATI-DME). Bogotá: Ministerio de Protección 
Social. Recuperado de https://www.minsalud. gov.co/Documentos\%20y\%20Publicaciones/ GATISO-DESORDENES\%20MUSCULARES\%20 ESQUELETICOS.pdf\#search=osteomusculares. Bogotá, Colombia.

Ministerio de la Protección Social. (2006). Guía de atención integral basada en la evidencia para Dolor Lumbar Inespecífico y Enfermedad Discal relacionados con la manipulación manual de cargas y otros factores de riesgo en el lugar de trabajo (GATIDLI- ED). Bogotá: Ministerio de la Protección Socia. Recuperado el 30 de noviembre de 2006, de https://www.minsalud.gov.co/Documentos\%20 y $\% 20$ Publicaciones/GATISO-DOLOR $\% 20$ L U M B A R \% 20 I N E S P E C \% C $3 \% 8$ D F I C O. pdf\#search=osteomusculares. Bogotá, Colombia.

NIOSH, I. N. (15 de diciembre de 2012). Acerca de NIOSH. HHS. Go-Departamento de Salud y Servicios Humanos, 1. Recuperado el 4 de abril de 2012, de GobiernoUSA.gov. Washington, D.C. Estados Unidos.

OIT E.P. (26 de abril de 2013). OIT urge una acción mundial para combatir las enfermedades profesionales. Recuperado de http://www.ilo.org/global/about-the-ilo/ newsroom/news/WCMS_211645/lang--es/index.htm:
Oficina Internacional del Trabajo (OIT). (2010). Programa de Seguridad y Salud en el Trabajo y Medio Ambiente (SafeWork). Recuperado de www.ilo.org/safework

\section{AUTORAS}

\section{Astrid Ordóñez}

Profesional de Salud Ocupacional de la Institución Universitaria Antonio José Camacho y miembro del semillero SERGOS de la misma institución. Correo: asorag@gmail.com

\section{Juliet Restrepo}

Terapeuta Ocupacional, Especialista en Ergonomía, Magister en Salud Ocupacional, Docente Tiempo Completo en la Institución Universitaria Antonio José Camacho. Integrante del Grupo de investigación GISAP. Correo: jcrestrepo@admon.uniajc.edu.co

\section{Claudia Castañeda}

Pregrado en Derecho, Magister en Educación, Directora del programa de Salud Ocupacional de la Institución Universitaria Antonio José Camacho. Integrante del Grupo de investigación GISAP. Correo: ccastaneda@admon.uniajc.edu.co 